\title{
A possible 3:2 orbital epicyclic resonance in QPO frequencies of Sgr A $^{*}$
}

\author{
G. Török ${ }^{1,2,3}$ \\ 1 Nordita, Blegdamsvej 17, 2100 Copenhagen, Denmark \\ 2 Department of Astrophysics, Göteborg University \& Theoretical Physics, Chalmers University, 412-96 Göteborg, Sweden \\ 3 Institute of Physics, Faculty of Philosophy and Science, Silesian University in Opava, Bezručovo Nám. 13, \\ 74601 Opava, Czech Republic \\ e-mail: terek@volny.cz
}

Received 17 December 2004 / Accepted 7 May 2005

\begin{abstract}
A recent measurement of double peak QPOs frequencies in Sgr A* is consistent with the 3:2 ratio. The same ratio is firmly established by previous observations in all double peaked $\mathrm{kHz}$ QPOs in microquasars and theoretically explained by orbital epicyclic resonances excited in nearly Keplerian accretion flow in a black hole's strong gravity. If confirmed, the 3:2 ratio of double peak QPOs in Sgr A* will be of a fundamental importance for the black hole accretion theory, by providing another clear argument that the accretion disk oscillations are indeed governed by non-linear, strong-gravity physics.
\end{abstract}

Key words. black hole physics - X-rays: individuals: Sgr A

\section{Double peak QPOs with the 3:2 ratio in Sgr $A^{*}$ ?}

From the current analysis of stellar orbits within 10-100 light hours of Sgr A*, obtained independently by the MPI Garching group (Schoedel et al. 2002, 2003; Eisenhauer et al. 2003) and the UCLA group (Ghez et al. 2003, 2004) the best estimate of the black hole central mass is $3.6 \pm 0.4 \times 10^{6} M_{\odot}$, where the error bars represent both statistical and systematic errors. Earlier lower statistical mass estimates based on proper motions of stars further away gave somewhat lower masses $\left(2.6 \times 10^{6} M_{\odot}\right)$ but in light of new information on stellar distribution and anisotropies these earlier data would now also lead to masses near $3.5 \times 10^{6} M_{\odot}$ (see the discussion in Schoedel et al. 2003). This well constrained mass must be contained within a few light hours, i.e. several hundred Schwarzschild radii. The analysis of the spatial distributiuon of the stellar cusp centered on the BH suggests that most likely no more than $1 \times 10^{3} M_{\odot}$ of that is in the form of stars or stellar remnants (the latter is less well constrained: Genzel et al. 2003). From the lack of motion of the radio source itself and a theoretical comparison of the stochastic motions of a BH of different masses with surrounding stars, a lower limit of the mass contained within the radius of the radio source (10 light min, 20 Schwarzschild radii) is about $\sim 10^{5} M_{\odot}$ (Reid et al. 1999, 2003; Backer \& Sramek 1999; Schoedel et al. 2003).
From these measurements and discussion, one concludes that the mass of the black hole in Sgr A* is most likely in the interval

$2.6 \times 10^{6} M_{\odot}<M<4.4 \times 10^{6} M_{\odot}$,

and that a very conservative lower limit is $\sim 10^{5} M_{\odot}{ }^{1}$.

Genzel et al. (2003) measured a clear periodicity of $17 \mathrm{~min}$ (1020 s) in Sgr A* variability during a flaring event. This period is in the range of Keplerian orbital periods at a few gravitational radii away from a black hole with the mass constrained by Eq. (1). More recently, Aschenbach et al. (2004) have reported three other QPOs periodicities, $692 \mathrm{~s}, 1130 \mathrm{~s}, 2178 \mathrm{~s}$, roughly in the orbital Keplerian range, and two much shorter periods of $100 \mathrm{~s}$ and $219 \mathrm{~s}$. The value of $1130 \mathrm{~s}$ differ by $10 \%$ from the $1020 \mathrm{~s}$ period found by Genzel et al. (2003) and may correspond to the same periodicity of the source, but a firm conclusion is not possible because of the quality of the data. With all reservations and caution that are necessary here, it was noticed (Abramowicz et al. 2004a,c; Aschenbach 2004) that

$(1 / 692):(1 / 1130):(1 / 2178) \approx 3: 2: 1$,

i.e. that the "Keplerian" frequencies found in Sgr A* form ratios that are very close to be an exact commensurable sequence,

1 We thank Reinhard Genzel for providing (in May 2004) this updated discussion on the Sgr A* mass measurements. 
Table 1. Frequencies of twin peak QPOs in microquasars and Galaxy centre black hole.

\begin{tabular}{l|cc|cc|c|c}
\hline \hline Source $^{a}$ & $v_{\text {upp }}[\mathrm{Hz}]$ & $\Delta v_{\text {upp }}[\mathrm{Hz}]$ & $v_{\text {down }}[\mathrm{Hz}]$ & $\Delta v_{\text {down }}[\mathrm{Hz}]$ & $2 v_{\text {upp }} / 3 v_{\text {down }}-1$ & Mass $^{b}\left[M_{\odot}\right]$ \\
\hline GRO 1655-40 & 450 & \pm 3 & 300 & \pm 5 & 0.00000 & $6.0-6.6$ \\
XTE 1550-564 & 276 & \pm 3 & 184 & \pm 5 & 0.00000 & $8.4-10.8$ \\
H 1743-322 & 240 & \pm 3 & 166 & \pm 8 & -0.03614 & not measured \\
GRS 1915+105 & 168 & \pm 3 & 113 & \pm 5 & 0.00885 & $10.0-18.0$ \\
\hline Sgr A & 1.445 & $\pm 0.16 \mathrm{mHz}$ & 0.886 & $\pm 0.04 \mathrm{mHz}$ & 0.08728 & $2.6-4.4 \times 10^{6}$ \\
\hline & \multicolumn{7}{|c}{}
\end{tabular}

a Twin peak QPOs first reported by Strohmayer (2001); Remillard et al. (2002); Homan et al. (2003); Remillard et al. (2003); Aschenbach et al. (2004).

${ }^{b}$ See Greene et al. (2001); Orosz et al. (2002); Greiner et al. (2001); McClintock \& Remillard (2003), and the first par of introduction.
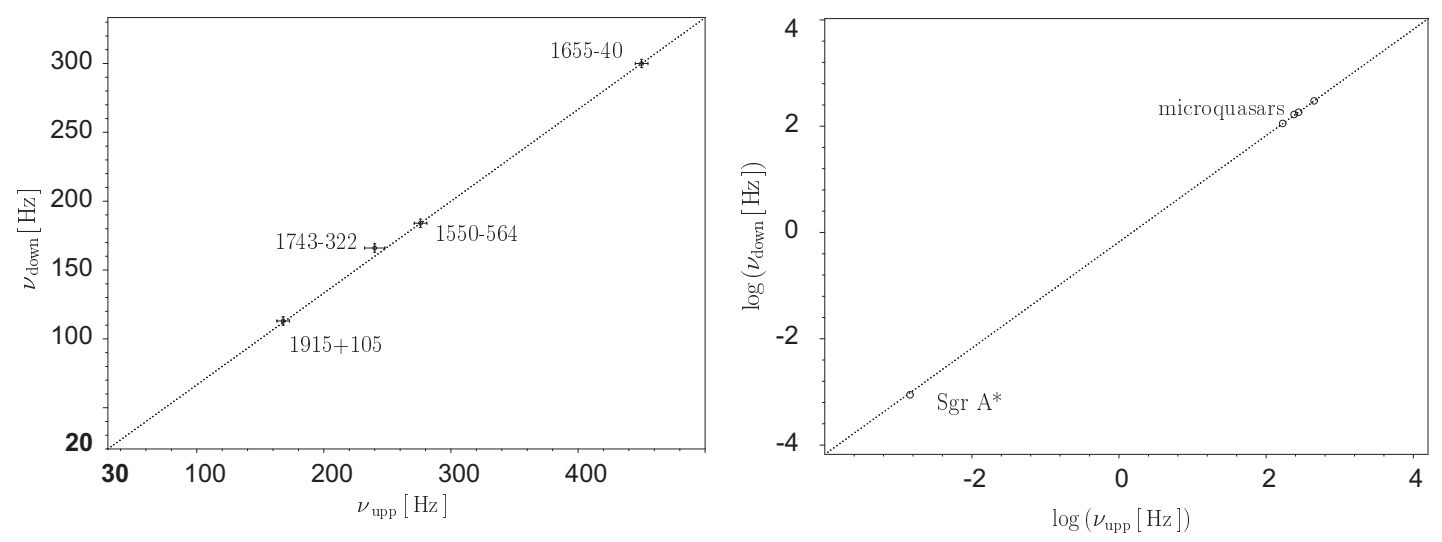

Fig. 1. Left: in all four microquasars where double peak $\mathrm{kHz}$ QPOs were detected, the observed frequencies $v_{\text {upp }}$ and $v_{\text {low }}$ are clearly in $3: 2$ ratio. Right: the same 3:2 ratio seems to be present in double peak QPOs in Sgr A*. The accuracy is so high that the error bars cannot be shown correctly in this logarithmic plot.

3:2:1. The commensurability of QPOs frequencies in Sgr A*, if confirmed by a more accurate observations and data analysis, could be of a fundamental importance for a reason that we explain in the next section.

\section{Commensurability of QPOs in microquasars: observations and theory}

In the case of stellar mass black hole sources, impressively accurate ratio of frequencies $v_{\text {upp }} / v_{\text {down }}=3 / 2$ was found in all four microquasars that display the double peak QPOs. Table 1 and Fig. 1 summarize the QPO microquasar data relevant to the present Note. In three microquasars with known mass $M$, the QPOs frequencies scale as (McClintock \& Remillard 2003),

$v_{\text {upp }}=2.8\left(\frac{M}{M_{\odot}}\right)^{-1}[\mathrm{kHz}]$.

Even before the double peak $\mathrm{kHz}$ QPOs were discovered in microquasars (first by Strohmayer 2001), and the 3:2 ratio pointed out (first by Abramowicz \& Kluźniak 2001), Kluźniak \& Abramowicz (2000) suggested on theoretical ground that these QPOs should have rational ratios, being due to resonances in oscillations of nearly Keplerian accretion disks. It seems that the resonance hypothesis is now well supported by observations, and that in particular the 3:2 ratio is seen most often in
Table 2. Relation of observed frequencies for standard $\left(v=v_{\mathrm{vert}}\right)$ and "Keplerian" $\left(v=v_{\mathrm{K}}\right)$ resonances.

\begin{tabular}{|c|c|c|c|c|c|}
\hline \multicolumn{4}{|c|}{ Theory } & \multicolumn{2}{|c|}{ Observed frequencies } \\
\hline \multicolumn{2}{|c|}{ Type of resonance } & \multicolumn{2}{|c|}{$n v_{\mathrm{rad}}=m v$} & \multirow[b]{2}{*}{$v_{\text {upp }}$} & \multirow[b]{2}{*}{$v_{\text {down }}$} \\
\hline & & $n$ & $m$ & & \\
\hline \multirow{3}{*}{ 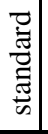 } & parametric & 3 & 2 & $v_{\text {vert }}$ & $v_{\mathrm{rad}}$ \\
\hline & $3: 1$ forced & 3 & 1 & $v_{\text {vert }}$ & $v_{\text {vert }}-v_{\text {rad }}$ \\
\hline & $2: 1$ forced & 2 & 1 & $v_{\mathrm{vert}}+v_{\mathrm{rad}}$ & $v_{\text {vert }}$ \\
\hline \multirow{3}{*}{ 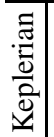 } & parametric & 3 & 2 & $v_{\mathrm{K}}$ & $v_{\mathrm{rad}}$ \\
\hline & $3: 1$ forced & 3 & 1 & $v_{\mathrm{K}}$ & $v_{\mathrm{K}}-v_{\mathrm{rad}}$ \\
\hline & $2: 1$ forced & 2 & 1 & $\nu_{\mathrm{K}}+v_{\mathrm{rad}}$ & $v_{\mathrm{K}}$ \\
\hline
\end{tabular}

double peak QPOs in LMXB black hole and neutron sources, $2 v_{\text {upp }}=3 v_{\text {down }}$.

According to the resonance hypothesis, the two modes in resonance have eigenfrequencies $v_{\mathrm{rad}}$, equal to the radial epicyclic frequency, and $v_{\mathrm{v}}$, equal to the vertical orbital frequency $v_{\text {vert }}$ or the Keplerian frequency $v_{\mathrm{K}}$ (see Kluźniak et al. 2004, for a recent review). Several resonances of this kind are possible, and have been discussed (see e.g. Abramowicz et al. 2004b). Main relations are summarized in Table 2. 
Table 3. Sgr A* spin estimates from observed 3:2 QPOs, calculated for five representative values of mass from a large spectrum above the lower conservative limit including the best mass estimate $3.6 \times 10^{6} M_{\odot}$.

\begin{tabular}{ll|lllllll}
\hline \hline & & \multicolumn{7}{|c}{ Mass $\left[M_{\odot}\right]:$} \\
\cline { 3 - 8 } Resonance & & $4.4 \times 10^{5}$ & $0.8 \times 10^{6}$ & $1.8 \times 10^{6}$ & $2.2 \times 10^{6}$ & $2.6 \times 10^{6}$ & $3.6 \times 10^{6}$ & $4.4 \times 10^{6}$ \\
\hline $3: 2\left[v_{\theta}, v_{\mathrm{r}}\right]$ & parametric & - & 0.22 & 0.90 & 0.98 & - & - & - \\
$2: 1\left[v_{\theta}, v_{\mathrm{r}}\right]$ & forced & - & - & 0.16 & 0.40 & 0.57 & 0.81 & 0.92 \\
$3: 1\left[v_{\theta}, v_{\mathrm{r}}\right]$ & forced & - & - & 0.36 & 0.58 & 0.72 & $0.95(0.99)^{*}$ & - \\
\hline $3: 2\left[v_{\mathrm{K}}, v_{\mathrm{r}}\right]$ & "Keplerian" p. & - & 0.25 & - & - & - & - & - \\
$2: 1\left[v_{\mathrm{K}}, v_{\mathrm{r}}\right]$ & "Keplerian" f. & - & - & 0.16 & 0.41 & 0.58 & 0.83 & 0.94 \\
$3: 1\left[v_{\mathrm{K}}, v_{\mathrm{r}}\right]$ & "Keplerian" f. & - & - & 0.32 & 0.52 & 0.65 & 0.85 & 0.93 \\
\hline
\end{tabular}

* See Fig. 2.
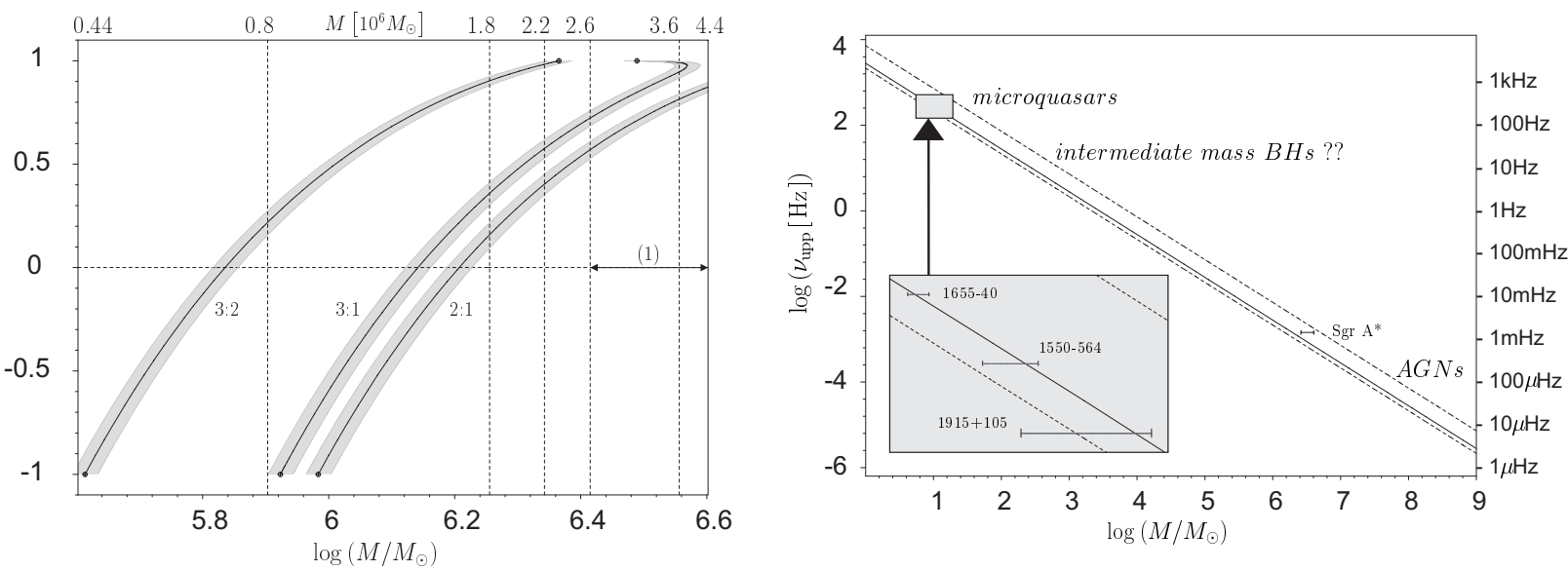

Fig. 2. Left: spin dependence for 3:2 parametric, 3:1 and 2:1 forced resonance in Sgr A* implied by measured $v_{\text {down }}=0.886 \mathrm{mHz}$, shadows respect accuracy of measuring. Right: the case of the 2:1 parametric resonance for observed frequencies. Mass range for Sgr A* corresponds to the range given by inequality (1). Dotted lines are for $a=0$ (lower line) and $a=1$ (upper line), the solid line is the best fit $v_{\text {upp vs. } 1 / M \text { found }}$ by McClintock \& Remillard (2003) and described by Eq. (3).

Formulae for $v_{\text {vert }}$ and $v_{\text {rad }}$ in the gravitational field of a rotating Kerr black hole with the mass $M$ and spin $a$ are well known,

$v_{\mathrm{vert}}^{2}=v_{\mathrm{K}}^{2}\left(1-4 a x^{-3 / 2}+3 a^{2} x^{-2}\right)$,

$v_{\mathrm{rad}}^{2}=v_{\mathrm{K}}^{2}\left(1-6 x^{-1}+8 a x^{-3 / 2}-3 a^{2} x^{-2}\right)$,

$v_{\mathrm{K}}=\frac{1}{2 \pi}\left(\frac{G M_{0}}{r_{G}^{3}}\right)^{1 / 2}\left(x^{3 / 2}+a\right)^{-1}$.

Here $x=r /\left(G M / c^{2}\right)$ is the dimensionless radius, expressed in terms of the gravitational radius of the black hole.

For a particular resonance $n: m$, the equation $n v_{\text {rad }}=m v$ $\left(v=v_{\mathrm{vert}}\right.$ or $\left.v_{\mathrm{K}}\right)$ determines the dimensionless resonance radius $x_{\mathrm{n}: \mathrm{m}}$ as a function of $a$.

\section{Application to Sgr $A^{*}$}

From the known mass of $\operatorname{Sgr} \mathrm{A}^{*}$, the observed $v_{\text {down }}=$ $0.886 \mathrm{mHz}=1 / 1130 \mathrm{~s}^{-1}$, and from Eq. (4) one may calculate the black hole spin in $\mathrm{SgrA}^{*}$, consistent with different types of resonances. This procedure was first applied to the microquasar GRO 1655-40 by Abramowicz \& Kluźniak (2001) and more recently for the other two microquasars by Abramowicz et al. (2004b) and Török et al. (2005). These results, together with these calculated in this Note for five representative values of the Sgr A* mass, are summarized in Table 3, and illustrated in Fig. 2 for particular resonances.

\section{Discussion and conclusions}

If commensurability of double peak QPOs frequencies in $\mathrm{Sgr} \mathrm{A}^{*}$ is confirmed, this together with the already established $1 / M$ scaling $^{2}$, would give very strong support for the suggestion that the double peak QPOs physics, in microquasars and in $\mathrm{Sgr} \mathrm{A}^{*}$, is due to a non-linear orbital resonance in strong gravity. It would be interesting to see whether other black hole sources, ULXs and AGNs, show the same phenomenon (Abramowicz et al. 2004a).

For black hole sources with known mass that display double peak QPOs, one may measure the black hole spin, but the spin estimate depends on which of the theoretically possible resonances, $2: 1,3: 1$, or $3: 2$, is actually excited in the source.

\footnotetext{
${ }^{2}$ See McClintock \& Remillard (2003); Kluźniak et al. (2004).
} 
At present, neither observations, nor the resonance theory can firmly determine this ${ }^{3}$.

Acknowledgements. I thank Marek Abramowicz and Wlodek Kluzniak and also Zdenek Stuchlik and Vladimir Karas for discussion and help. The article was partialy written under the Erasmus-Socrates exchange program between Chalmers University and Silesian University at Opava. The final version was realized with the support of Czech grant MSM 4781305903 and completed at Nordita (Copenhagen).

\section{References}

Abramowicz, M. A., \& Kluźniak, W. 2001, A\&A, 374, L19

Abramowicz, M. A., Kluźniak, W., McClintock, J. E., \& Remillard, R. A. 2004a, ApJ, 609, L63

Abramowicz, M. A., Kluźniak, W., Stuchlík, Z., \& Török, G. 2004b, A\&A, submitted [arXiv: astro-ph/0401464]

Abramowicz, M. A., \& Kluźniak, W. 2004, X-Ray Timing 2003: Rossi and Beyond, ed. P. Kaaret, F. K. Lamb, \& J. H. Swank (Melville, NY: American Institute of Physics)

Abramowicz, M. A., Kluźniak, W., Stuchlík, Z., \& Török, G. 2004c, in Proceedings of RAGtime 4/5: Workshops on black holes and neutron stars, Opava, 1416/1315 October 2002/03, Opava, 2004, Silesian University at Opava, ed. S. Hledík, \& Z. Stuchlík

Aschenbach, B., Grosso, N., Porquet, D., \& Predehl, P. 2004, A\&A, 417, 71

Aschenbach, B. 2004, A\&A, 425, 1075

Backer, D. C., \& Sramek, R. A. 1999, ApJ, 524, 805

Eisenhauer, F., Schödel, R., Genzel, R., et al. 2003, ApJ, 597, L121

Genzel, R., Schödel, R., Ott, T., et al. 2003, ApJ, 594, 812

Ghez, A. M., Duchêne, G., Matthews, K., et al. 2003, ApJ, 586, L127
Ghez, A. M., Salim, S., Hornstein, S. D., et al. 2004 [arXiv: astro-ph0306130]

Greene, J., Bailyn, Ch. D., \& Orosz, J. A. 2001, ApJ, 554, 1290

Greiner, J., Cuby, J. G., \& McCaughrean, M. J. 2001, Nature, 414, $522 \mathrm{G}$

Homan, J., Miller, J. M., Wijnands, R., et al. 2003, Atel 16 http://integral.rssi.ru/atelmirror/

Kluźniak, W., \& Abramowicz, M. A. 2000, Phys. Rev. Lett., submitted [arXiv:astro-ph/0105057]

Kluźniak, W., \& Abramowicz, M. A. 2003, 12th Workshop on General Relativity and Gravitation (Tokyo: Tokyo University Press) [arXiv: astro-ph/0304345]

Kluźniak, W., Abramowicz, M. A., \& Lee, W. H. 2004, in X-Ray Timing 2003: Rossi and Beyond, ed. P. Kaaret, F. K. Lamb, \& J. H. Swank (Melville, NY: American Institute of Physics)

McClintock, J. E., \& Remillard, R. A. 2003 [arXiv: astro-ph/0306213v.2]

Orosz, J. A., Groot, P. J., van der Klis, M., et al. 2002, ApJ, 568, 845

Remillard, R. A., Muno, M. P., McClintock, J. E., \& Orosz, J. A. 2002, ApJ, 580, 1030

Remillard, R. A., Muno, M. P., McClintock, J. E., \& Orosz, J. A. 2003, HEAD....7.3003

Reid, M. J., Menten, K. M., Genzel, R., et al. 1999, ApJ, 524, 816

Reid, M. J., Menten, K. M., Genzel, R., et al. 2003, Astron. Nachr./AN 324,505

Schoedel, R., Ott, T., Genzel, R., et al. 2002, Nature, 419, 694

Schoedel, R. Ott, T., Genzel, R., et al. 2003, ApJ, 596, 1015

Stuchlik, Z., Slany, P., Török, G., \& Abramowicz, M. A. 2004, Phys. Rev. D., submitted, [gr-qc/0411091]

Strohmayer, T. 2001, ApJ, 552, L49

Török, G., Abramowicz, M. A., Kluźniak, W., \& Stuchlík, Z. 2005, A\&A, 436, 1

\footnotetext{
${ }^{3}$ Aschenbach (2004) argues that QPOs data suggest that black holes in the three microquasars and Sgr A* listed in Table 1 have nearly the same spin $a \approx 0.99616$, due to a new relativistic effect that he found (a non monotonic behaviour of orbital velocity with radius for rapidly rotating black holes, see also Stuchlik et al. 2004). Our calculations are based on standard types of non-linear orbital resonances, and do not include the Aschenbach effect.
} 